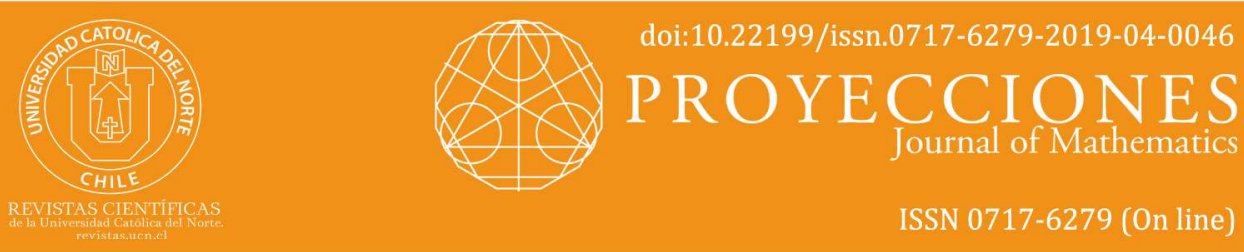

\title{
Some pairwise weakly fuzzy mappings
}

R. Dhar

"Maharaja Bir Bikram Univesity, Dept. of Applied Mathematics, Agartala, TR, India.

$\nabla$ runu.dhar@gmail.com

Received: June 2018 | Accepted: January 2019

\section{Abstract:}

The aim of this paper is to introduce some pairwise weakly fuzzy mappings, called pairwise weakly fuzzy $\delta$-semi-pre-continuous mappings and pairwise weakly fuzzy $\delta$-semi pre-open mappings in fuzzy bitopological spaces. The concept of pairwise weakly fuzzy $\delta$-semi-precontinuous mappings is to be introduced in fuzzy bitopological spaces with the help of the concept of (i,j)-fuzzy pre-open and (i, j)-fuzzy $\delta$-semi pre-open set. Then some of its basic properties and characterization theorems are to be investigated. Also the notion of pairwise weakly fuzzy $\delta$-semi-pre-open mappings is to be introduced in fuzzy bitopological spaces with the help of the concept of $(i, j)$-fuzzy open set and $(i, j)$-fuzzy $\delta$-semi preinterior. Some of its basic properties and its relationship with other known mappings are also to be studied.

Keywords: Fuzzy bitopological space; Pairwise weakly fuzzy; Fuzzy $\delta$-semi pre-continuous; Fuzzy $\delta$-semi pre-open mappings.

MSC (2010): 54A40.

\section{Cite this article as (IEEE citation style)}

R. Dhar, "Some pairwise weakly Fuzzy mappings", Proyecciones (Antofagasta, On line), vol. 38, no. 4, pp. 707-717, Oct. 2019, doi: 10.22199/issn.0717-6279-2019-04-0046. [Accessed dd-mm-yyyy].

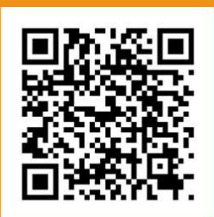

Article copyright: (C) 2019 Runu Dhar. This is an open access article distributed under the terms of the Creative Commons Licence, which permits unrestricted use and distribution provided the original author and source are credited. 


\section{Introduction}

Zadeh [25] introduced the fundamental concept of fuzzy sets in his classical paper. Chang [4] introduced the concept of fuzzy topological spaces as a generalization of topological spaces. Since then many topologists have contributed to the theory of fuzzy topological spaces. Thakur and Singh [21] introduced the concept of fuzzy semi pre-open sets and fuzzy semi precontinuity. Ganguly and Saha [7] introduced the concept of $\delta$-continuity and $\delta$-connected set in fuzzy set theory. The concept of weakly fuzzy $\delta$ semi-pre-continuous mappings and pairwise weakly fuzzy $\delta$-semi pre-open mappings in fuzzy topological spaces were studied by Mukherjee and Dhar $[12,13]$. Dutta and Tripathy [6] introduced fuzzy $b-\theta$-open sets in fuzzy topological spaces. Also Tripathy and Ray $[22,23]$ introduced $\delta$-continuity and weakly continuous functions on mixed fuzzy topological spaces. Dubey, Panwar and Tiwari [5] introduced weakly pairwise irresolute mappings. Kandil [8] introduced and studied the notion of fuzzy bitopological spaces (a system $\left(X, \tau_{1}, \tau_{2}\right)$ consisting of a non-empty set $\mathrm{X}$ with two arbitrary topologies $\tau_{1}$ and $\tau_{2}$ on $\mathrm{X}$ is called a fuzzy bitopological space) as a natural generalization of fuzzy topological spaces. Also D. Sarma and B.C. Tripathy [17] and B.C. Tripathy and S. Debnath [24] introduced and studied different concepts in bitopological spaces. In this paper, the concept of weakly fuzzy $\delta$-semi- pre-continuous mappings and pairwise weakly fuzzy $\delta$-semi pre-open mappings in fuzzy bitopological spaces are to be introduced. Throughout the present study, the spaces $X, Y$ and $Z$ always represent fuzzy bitopological spaces $\left(X, \tau_{1}, \tau_{2}\right),\left(Y, \sigma_{1}, \sigma_{2}\right)$ and $\left(Z, \Gamma_{1}, \Gamma_{2}\right)$ respectively. As to the notations and terminologies $c l(A)$ and $\operatorname{int}(A)$ to be denote the closure of $A$ and the interior of $A$, respectively in a fuzzy topological space $(X, \tau)$. Also $\tau_{i}-\operatorname{int}(\lambda)$ and $\tau_{j}-c l(\lambda)$ to be mean respectively the interior and closure of a fuzzy set $\lambda$ with respect to the fuzzy topologies $\tau_{i}$ and $\tau_{j}$ in a fuzzy bitopological space $\left(X, \tau_{1}, \tau_{2}\right)$.

\section{Preliminaries and Definitions}

In this section, some preliminary results and definitions have been procured.

Definition 2.1. [25] Let $X$ be a crisp set and $A$ and $B$ be two fuzzy subsets of $X$ with membership functions $\mu_{A}$ and $\mu_{B}$ respectively. Then

(a) $A$ is equal to $B$, i.e., $A=B$ if and only if $\mu_{A}(x)=\mu_{B}(x)$, for all $x \in X$,

(b) $A$ is called a subset of $B$ if and only if $\mu_{A}(x) \leq \mu_{B}(x)$, for all $x \in X$,

(c) the Union of two fuzzy sets $A$ and $B$ is denoted by $A \vee B$ and its 
membership function is given by $\mu_{A \vee B}=\max \left(\mu_{A}, \mu_{B}\right)$,

(d) the Intersection of two fuzzy sets $A$ and $B$ is denoted by $A \wedge B$ and its membership function is given by $\mu_{A \wedge B}=\min \left(\mu_{A}, \mu_{B}\right)$,

(e) the Complement of a fuzzy set $A$ is defined as the negation of the specified membership function. Symbolically it can be written as $\mu_{A}^{c}=$ $1-\mu_{A}$.

Definition 2.2. [16] A fuzzy point $x_{p}$ in $X$ is a fuzzy set in $X$ defined by

$$
x_{p}(y)= \begin{cases}p(0<p \leq 1), & \text { for } y=x \\ 0, & \text { for } y \neq x(y \in X) .\end{cases}
$$

$x$ and $p$ are respectively the support and the value of $x_{p}$.

A fuzzy point $x_{p}$ is said to belong to a fuzzy set $A$ of $X$ if and only if $p \leq A(x)$. A fuzzy set $A$ in $X$ is the union of all fuzzy points which belong to $A$.

Definition 2.3. [3] Let $A$ be a fuzzy subset of a fuzzy topological space $(X, \tau)$. Then $A$ is called fuzzy $\delta$-pre-open if $A \leq \operatorname{int}(\delta c l(A))$. The complement of a fuzzy $\delta$-pre-open set is called fuzzy $\delta$-pre-closed .

Definition 2.4. A fuzzy subset $\lambda$ in a fuzzy topological space $X$ is called

(a) [1] fuzzy semi-open if $\lambda \leq \operatorname{cl}(\operatorname{int}(\lambda))$

(b) [2] fuzzy pre-open if $\lambda \leq \operatorname{int}(\operatorname{cl}(\operatorname{int}(\lambda)))$

(c) [21] fuzzy semi pre-open if there exists a fuzzy pre-open set $\mu$ such that $\mu \leq \lambda \leq c l(\mu)$.

Definition 2.5. [18] A fuzzy subset $\gamma$ in $(X, \tau)$ is said to be fuzzy $\delta$-semi pre-open if there exists a fuzzy $\delta$-pre-open set $\mu$ such that $\mu \leq \gamma \leq \delta-\operatorname{cl}(\mu)$ or equivalently $\gamma \leq \delta-\operatorname{cl}($ int $\delta-\operatorname{cl}(\gamma))$.

Definition 2.6. [1] A fuzzy subset $A$ of a fuzzy topological space $(X, \tau)$ is called

(a) a fuzzy regular open set of $(X, \tau)$ if int $\operatorname{cl}(A)=A$ and

(b) a fuzzy regular closed set of $(X, \tau)$ if $\operatorname{cl} \operatorname{int}(A)=A$.

Definition 2.7. [14] A fuzzy subset $A$ of a fuzzy topological space $(X, \tau)$ is said to be fuzzy $\delta$-semi-open if $A \leq \operatorname{clint}_{\delta}(A)$. 
Definition 2.8. [11] A mapping $f:(X, \tau) \rightarrow(Y, \sigma)$ from a fuzzy topological space $(X, \tau)$ to another fuzzy topological space $(Y, \sigma)$ is said to be weakly fuzzy $\delta$-semi-pre-continuous mapping if $f^{-1}(\alpha) \in f \delta$ spo $(X)$ for each $\alpha \in f p o(Y)$, where $f \delta s p o(X)$ (respectively $f p o(X)$ ) denotes the family of all fuzzy $\delta$-semi pre-open (respectively fuzzy pre-open) sets of $X$.

Definition 2.9. [12] The function $f:(X, \tau) \rightarrow(Y, \sigma)$ is said to be weakly fuzzy $\delta$-semi pre-open if $f(\lambda) \leq \delta \operatorname{spint}(f p c l \lambda)$ for each fuzzy open set $\lambda$ of $X$.

Definition 2.10. [12] A function $f:(X, \tau) \rightarrow(Y, \sigma)$ is said to be weakly fuzzy $\delta$-semi pre-closed if $\delta \operatorname{spcl}(f(\operatorname{pint}(\lambda))) \leq f(\lambda)$ for each fuzzy pre-closed subset $\lambda$ of $X$.

Definition 2.11. [10] Let $\left(X, \tau_{1}, \tau_{2}\right)$ be a fuzzy bitopological space. The $(i, j)$ - fuzzy semi-closure (denoted by $(i, j)$-scl $(A))$ and $(i, j)$-fuzzy semiinterior (denoted by $(i, j)$-sint $(A))$ of a fuzzy set $A$ in $\left(X, \tau_{1}, \tau_{2}\right)$ are defined respectively as follows :

(a) $(i, j)-\operatorname{scl}(A)=\inf \{B: B \geq A, B$ is $(i, j)$-fuzzy semi-closed $\}$,

(b) $(i, j)-\operatorname{sint}(A)=\sup \{B: B \leq A, B$ is $(i, j)$-fuzzy semi-open $\}$.

Definition 2.12. Let $f:\left(X, \tau_{1}, \tau_{2}\right) \rightarrow\left(Y, \sigma_{1}, \sigma_{2}\right)$ be a mapping from a fuzzy bitopological space $\left(X, \tau_{1}, \tau_{2}\right)$ to another fuzzy bitopological space $\left(Y, \sigma_{1}, \sigma_{2}\right)$. Then $f$ is called

(a) [19] pairwise fuzzy continuous (respectively pairwise fuzzy open) if $f:\left(X, \tau_{1}\right) \rightarrow\left(Y, \sigma_{1}\right)$ and $f:\left(X, \tau_{2}\right) \rightarrow\left(Y, \sigma_{2}\right)$ are fuzzy continuous (respectively fuzzy open),

(b) [20] pairwise fuzzy irresolute if the inverse image of each $(i, j)$-fuzzy semi-open set in $Y$ is $(i, j)$-fuzzy semi-open set in $X, i \neq j, i, j=1,2$,

(c) [10] pairwise fuzzy semi-continuous if the inverse image of each $\sigma_{i}$-fuzzy open set in $Y$ is $(i, j)$-fuzzy semi-open set in $X, i \neq j, i, j=1,2$,

(d) [10] pairwise fuzzy semi-open if the image of every $\tau_{i}$-fuzzy open set in $X$ is $(i, j)$-fuzzy semi-open set in $Y, i \neq j, i, j=1,2$,

(e) [9] pairwise fuzzy pre-continuous if the inverse image of each $\sigma_{i}$-fuzzy open set in $Y$ is $(i, j)$-fuzzy pre-open set in $X, i \neq j, i, j=1,2$,

(f) [9] pairwise fuzzy pre-open if the image of every $\tau_{i}$-fuzzy open set in $X$ is $(i, j)$-fuzzy pre-open set in $Y, i \neq j, i, j=1,2$,

(g) [15] pairwise fuzzy semi-pre-continuous if the inverse image of each $\sigma_{i^{-}}$ fuzzy open set in $Y$ is $(i, j)$-fuzzy semi pre-open set in $X, i \neq j, i, j=1,2$,

(h) [15] pairwise fuzzy semi pre-open if the image of every $\tau_{i}$-fuzzy open set in $X$ is $(i, j)$-fuzzy semi pre-open set in $Y, i \neq j, i, j=1,2$. 
Definition 2.13. [13] A subset $A$ of a fuzzy bitopological space $\left(X, \tau_{1}, \tau_{2}\right)$ is said to be $(i, j)$-fuzzy $\delta$-semi-open if $A \leq \tau_{j}-c l\left(\tau_{i}-i n t_{\delta}(A)\right)$. The complement of $(i, j)$-fuzzy $\delta$-semi-open set is called $(i, j)$-fuzzy $\delta$-semi-closed.

Definition 2.14. [13] Let $A$ be a subset of a fuzzy bitopological space $\left(X, \tau_{1}, \tau_{2}\right)$. Then

(a) the intersection of all $(i, j)$-fuzzy $\delta$-semi-closed sets containing $A$ is called the $(i, j)$-fuzzy $\delta$-semi-closure of $A$ and is denoted by $(i, j)$-scl $\delta_{\delta}(A)$,

(b) the union of all $(i, j)$-fuzzy $\delta$-semi-open sets contained in $A$ is called the $(i, j)$-fuzzy $\delta$-semi-interior of $A$ and is denoted by $(i, j)-\operatorname{sint}_{\delta}(A)$.

Definition 2.15. [13] A subset $A$ of a fuzzy bitopological space $\left(X, \tau_{1}, \tau_{2}\right)$ is said to be $(i, j)$-fuzzy $\delta$-semi pre-open if $A \leq\left((i, j)-\operatorname{spint}_{\delta}(i, j)-\operatorname{spcl}_{\delta}(A)\right)$. The complement of $(i, j)$-fuzzy $\delta$-semi pre-open set is called $(i, j)$-fuzzy $\delta$ semi pre-closed.

Definition 2.16. [13] Let $A$ be a subset of a fuzzy bitopological space $\left(X, \tau_{1}, \tau_{2}\right)$. Then

(a) the intersection of all $(i, j)$-fuzzy $\delta$-semi pre-closed sets containing $A$ is called the $(i, j)$-fuzzy $\delta$-semi pre-closure of $A$ and is denoted by $(i, j)$ $\operatorname{spcl}_{\delta}(A)$,

(b) the union of all $(i, j)$-fuzzy $\delta$-semi pre-open sets contained in $A$ is called the $(i, j)$-fuzzy $\delta$-semi pre-interior of $A$ and is denoted by $(i, j)$-spint ${ }_{\delta}(A)$.

\section{Pairwise weakly fuzzy $\delta$ - semi precontinuous mappings}

In this section the concept of weakly fuzzy $\delta$-semi-pre-continuous mapping is introduced. Some characterization theorems and its basic properties are studied.

Definition 3.1. For any two fuzzy bitopological spaces $\left(X, \tau_{1}, \tau_{2}\right)$ and $\left(Y, \sigma_{1}, \sigma_{2}\right)$, a mapping $f: X \rightarrow Y$ is said to be pairwise weakly fuzzy $\delta$-semi-pre-continuous if for each $x_{p}$ in $X$ and each $(i, j)$-fuzzy- $\delta$-semi preopen set $V$ containing $f\left(x_{p}\right)$, there is an $(i, j)$-fuzzy pre-open set $U$ in $X$ such that $x_{p} \in U$ and $f(U) \leq(i, j)-\operatorname{spcl}_{\delta}(V), i \neq j$ and $i, j=1,2$.

Theorem 3.2. For any mapping $f:\left(X, \tau_{1}, \tau_{2}\right) \rightarrow\left(Y, \sigma_{1}, \sigma_{2}\right)$, the following conditions are equivalent:

(i) For any subset $A$ of $\left.\left.Y,(i, j)-\operatorname{spcl}_{\delta}\left(f^{-1}(i, j)-\operatorname{spint}_{\delta}(i, j) \operatorname{spcl}_{\delta}(A)\right)\right)\right) \leq$ $f^{-1}\left((i, j)-\operatorname{spcl}_{\delta}(A)\right)$. 
(ii) For any $(i, j)$ - fuzzy pre-open set $G$ in $Y,(i, j)-\operatorname{spcl}_{\delta}\left(f^{-1}(G)\right) \leq$ $f^{-1}\left((i, j)-\operatorname{spcl}_{\delta}(G)\right)$.

(iii) For any $(i, j)$ - fuzzy pre-open set $H$ in $G$ in $Y,(i, j)-\operatorname{spcl}_{\delta}\left(f^{-1}(i, j)\right)$ spint $\left._{\delta}(H)\right) \leq f^{-1}(H)$, where $i \neq j$ and $i, j=1,2$.

Proof. $\quad(\mathrm{i}) \Rightarrow($ ii). Let $G$ be any $(i, j)$-fuzzy pre-open set in $Y$. Then, by (i), $\left.\left.(i, j)-\operatorname{spcl}_{\delta}\left(f^{-1}(i, j)-\operatorname{spint}_{\delta}(i, j)-\operatorname{spcl}_{\delta}(G)\right)\right)\right) \leq f^{-1}\left((i, j)-\operatorname{spcl}_{\delta}(G)\right)$. Since $G$ is $(i, j)$-fuzzy pre-open, $G \leq\left((i, j)-\operatorname{spint}_{\delta}(i, j)-\right.$ spcl $\left._{\delta}(G)\right)$.Consequently, $(i, j)-\operatorname{spcl}_{\delta}\left(f^{-1}(G)\right) \leq f^{-1}\left((i, j)-\operatorname{spcl}_{\delta}(G)\right)$.

(ii) $\Rightarrow$ (iii). For any $(i, j)$-fuzzy $\delta$-semi pre-closed set $H$ in $Y,(i, j)$ $\operatorname{spint}_{\delta}(H)$ is $(i, j)$-fuzzy $\delta$ - semi pre-open set in $Y$. Therefore, by (ii), $(i, j)$ $\left.\operatorname{spcl}_{\delta}\left(f^{-1}(i, j)-\operatorname{spint}_{\delta}(H)\right) \leq f^{-1}\left((i, j)-\operatorname{spcl}_{\delta}(i, j)-\operatorname{spint}_{\delta}(H)\right)\right)$. Since $H$ is $(i, j)$-fuzzy $\delta$-semi pre-closed, $(i, j)$-spcl $((i, j)$-spint $\delta(H)) \leq H$. Therefore, $\left.(i, j)-\operatorname{spcl}_{\delta}\left(f^{-1}(i, j)\right)-\operatorname{spint}_{\delta}(H)\right) \leq f^{-1}(H)$.

(iii) $\Rightarrow$ (i). Let $A$ be any fuzzy subset of $Y$. Let $H=(i, j)-\operatorname{spcl}_{\delta}(A)$. Then for the $(i, j)$-fuzzy $\delta$-semi pre-closed set $H$, by (iii), $(i, j)-\operatorname{spcl}_{\delta}\left(f^{-1}(i, j)\right.$ $\left.\left.\left.\operatorname{spint}_{\delta}(i, j)-\operatorname{spcl}_{\delta}(A)\right)\right)\right) \leq f^{-1}\left((i, j)-\operatorname{spcl}_{\delta}(A)\right)$.

Theorem 3.3. A mapping $f:\left(X, \tau_{1}, \tau_{2}\right) \rightarrow\left(Y, \sigma_{1}, \sigma_{2}\right)$ is pairwise weakly fuzzy $\delta$-semi-pre -continuous if and only if for any $(i, j)$-fuzzy pre-open set $V$ in $Y, f^{-1}(V) \leq(i, j)-\operatorname{spint}_{\delta}\left(f^{-1}\left((i, j)-\operatorname{spcl}_{\delta}(V)\right), i \neq j\right.$ and $i, j=1,2$.

Proof. Let $f$ be pairwise weakly fuzzy $\delta$ - semi-pre-continuous mapping and let $V$ be any $(i, j)$-fuzzy pre-open set in $Y$. Then for any $x_{p} \in X$ with $x_{p} \in f^{-1}(V)$, there exists some $(i, j)$-fuzzy $\delta$ - semi pre-open set $U$ in $X$ such that $x_{p} \in U$ and $f(U) \leq\left((i, j)-\operatorname{spcl}_{\delta}(V)\right.$. Hence $x_{p} \in U \leq$ $f^{-1}\left((i, j)-\operatorname{spcl}_{\delta}(V)\right)$. Consequently, $x_{p} \in(i, j)-\operatorname{spint}_{\delta}\left(f^{-1}(i, j)-\operatorname{spcl}_{\delta}(V)\right)$ and $f^{-1}(V) \leq(i, j)-\operatorname{spint}_{\delta}\left(f^{-1}\left((i, j)-\operatorname{spcl}_{\delta}(V)\right)\right.$.

Conversely, let $x_{p} \in X$ and $V$ be any $(i, j)$ - fuzzy pre-open set in $Y$ with $f\left(x_{p}\right) \in V$. Then by hypothesis $f^{-1}(V) \leq(i, j)$ spint $_{\delta}\left(f^{-1}((i, j)\right.$ $\left.\operatorname{spcl}_{\delta}(V)\right)$. Put $U=(i, j)-\operatorname{spint}_{\delta}\left(f^{-1}\left((i, j)-\operatorname{spcl}_{\delta}(V)\right)\right.$. Then $(i, j)$-fuzzy $\delta$ -semi pre-open subset $U$ is such that $x_{p} \in U \leq f^{-1}\left((i, j)-\operatorname{spcl}_{\delta}(V)\right)$. Therefore, $f(U) \leq(i, j)-s p c l_{\delta}(V)$.

Theorem 3.4. A mapping $f:\left(X, \tau_{1}, \tau_{2}\right) \rightarrow\left(Y, \sigma_{1}, \sigma_{2}\right)$ is pairwise weakly fuzzy $\delta$ - semi-pre-continuous if and only if for any fuzzy subset $A$ of $Y$, $(i, j)-\operatorname{spcl}_{\delta}\left(f^{-1}(i, j)-\operatorname{spint}_{\delta}\left(\left((i, j)-\operatorname{spcl}_{\delta}(A)\right)\right)\right) \leq f^{-1}\left((i, j)-\operatorname{spcl}_{\delta}(A)\right), i \neq j$ and $i, j=1,2$. 
Proof. Sufficiency. Let $A$ be any subset of $Y$ and $x_{p} \in X$ be such that $x_{p} \notin f^{-1}\left((i, j)-\operatorname{spcl}_{\delta}(A)\right)$. Then $f\left(x_{p}\right) \notin f^{-1}\left((i, j)-\operatorname{spcl}_{\delta}(A)\right)$ and so there exists some $(i, j)$-fuzzy pre-open set $W$ in $Y$ such that $f\left(x_{p}\right) \in W$ and $W \cap A=0_{X}$. Since $f$ is pairwise weakly fuzzy $\delta$-semi-pre -continuous, there exists some $(i, j)$ - fuzzy $\delta$ - semi pre-open set $U$ in $X$ such that $x_{p} \in U$ and $f(U) \leq(i, j)-\operatorname{spcl}_{\delta}(W)$. Further, $W \cap(i, j)-\operatorname{spcl}_{\delta}(A)=0_{X}$ and $(i, j)-\operatorname{spcl}_{\delta}\left(Y-(i, j)-\operatorname{spcl}_{\delta}(A)\right)=\left(Y-(i, j)-\operatorname{spint}_{\delta}\left(\left((i, j)-\operatorname{spcl}_{\delta}(A)\right)\right.\right.$. Therefore, $f(U) \leq\left(Y-(i, j)-\right.$ spint $_{\delta}\left((i, j)-\right.$ spcl $\left._{\delta}(A)\right)$ and hence, $f(U) \cap$ $(i, j)-\operatorname{spint}_{\delta}(i, j)-\operatorname{spcl}_{\delta}(A)=0_{X}$. Consequently, $U \cap f^{-1}\left((i, j)-\operatorname{spint}_{\delta}(i, j)-\right.$ $\left.\operatorname{spcl}_{\delta}(A)\right)=0_{X}$. It follows that $x_{p} \notin(i, j)-\operatorname{spcl}_{\delta}\left(f^{-1}(i, j)-\operatorname{spint}_{\delta}(i, j)-\right.$ $\left.\left.\operatorname{spcl}_{\delta}(A)\right)\right)$. Hence $(i, j)-\operatorname{spcl}_{\delta}\left(f^{-1}(i, j)-\operatorname{spint}_{\delta}\left(\left((i, j)-\operatorname{spcl}_{\delta}(A)\right)\right)\right) \leq f^{-1}((i, j)-$ $\left.\operatorname{spcl}_{\delta}(A)\right)$.

Necessity. Let $x_{p} \in X$ and $V$ be any $(i, j)$-fuzzy pre-open set in $Y$ with $f\left(x_{p}\right) \in V$. Then $V \cap(Y-(i, j)$-spcl $-\delta(V))=0_{X}$. Therefore, $f\left(x_{p}\right) \notin$ $(i, j)-\operatorname{spcl}_{\delta}\left(Y-(i, j)-\operatorname{spcl}_{\delta}(V)\right)$ and hence $x_{p} \notin f^{-1}\left((i, j)-\operatorname{spcl}_{\delta}(Y-(i, j)-\right.$ $\left.\left.\operatorname{spcl}_{\delta}(V)\right)\right)$. Now, $(Y-(i, j)-\operatorname{spcl}-\delta(V)) \leq(i, j)-\operatorname{spint}_{\delta}(i, j)-\operatorname{spcl}_{\delta}(Y-(i, j)-$ $\left.\left.\operatorname{spcl}_{\delta}(V)\right)\right)$ and by hypothesis, $(i, j)-\operatorname{spcl}_{\delta}\left(f^{-1}\left((i, j)-\operatorname{spint}_{\delta}\left((i, j)-\operatorname{spcl}_{\delta}(Y-\right.\right.\right.$ $\left.\left.\left.(i, j)-\operatorname{spcl}_{\delta}(V)\right)\right)\right) \leq f^{-1}(i, j)-\operatorname{spcl}_{\delta}\left(Y-(i, j)-\operatorname{spcl}_{\delta}(V)\right)$. Therefore, $x_{p} \notin$ $\left((i, j)-\operatorname{spcl}_{\delta}\left(f^{-1}\left(Y-(i, j)-\operatorname{spcl}_{\delta}(V)\right)\right)\right)$. Therefore, there exists some $(i, j)$ fuzzy- $\delta$-semi pre-open set $U$ in $X$ such that $x_{p} \in U$ and $U \cap f^{-1}((i, j)$ $\left.\operatorname{spcl}_{\delta}(V)\right)=0_{X}$. Consequently, $U \leq X-f^{-1}\left(Y-(i, j)-\operatorname{spcl}_{\delta}(V)\right)=f^{-1}((i, j)-$ $\left.\operatorname{spcl}_{\delta}(V)\right)$. Therefore, it follows that $f(U) \leq(i, j)-\operatorname{spcl}_{\delta}(V)$.

Theorem 3.5. Let $\left(X, \tau_{1}, \tau_{2}\right)$ and $\left(Y, \sigma_{1}, \sigma_{2}\right)$ be any two fuzzy bitoplogical spaces and let $f:\left(X, \tau_{1}, \tau_{2}\right) \rightarrow\left(Y, \sigma_{1}, \sigma_{2}\right)$ be a mapping. Then the following statements are equivalent:

(i) The mapping $f$ is pairwise weakly fuzzy $\delta$-semi-pre-continuous.

(ii) For each $\left.\left.A \leq Y,(i, j)-\operatorname{spcl}_{\delta}\left(f^{-1}(i, j)-\operatorname{spint}_{\delta}(i, j)-\operatorname{spcl}_{\delta}(A)\right)\right)\right) \leq f^{-1}((i, j)$ $\left.\operatorname{spcl}_{\delta}(A)\right)$.

(iii) For each $(i, j)$-fuzzy pre-open set $G$ in $Y$, $(i, j)-\operatorname{spcl}_{\delta}\left(f^{-1}(G)\right) \leq$ $f^{-1}\left((i, j)-\operatorname{spcl}_{\delta}(G)\right)$.

(iv) For each $(i, j)$-fuzzy pre-open set $G$ in $Y,(i, j)-\operatorname{spcl}_{\delta}\left(f^{-1}\left((i . j)-\operatorname{spint}_{\delta}(H)\right)\right) \leq$ $f^{-1}(H)$.

(v) For each $(i, j)$-fuzzy pre-open set $G$ in $Y, f^{-1}(G) \leq\left((i . j)-\right.$ spint $_{\delta}\left(f^{-1}((i, j)\right.$ $\left.\left.\operatorname{spcl}_{\delta}(G)\right)\right)$ where $i \neq j$ and $i, j=1,2$.

Proof. The proof follows from the Theorem 3.2., Theorem 3.3. and Theorem 3.4. and hence omitted. 
Theorem 3.6. For any three fuzzy bitopological spaces $\left(X, \tau_{1}, \tau_{2}\right),\left(Y, \sigma_{1}, \sigma_{2}\right)$ and $\left(Z, \Gamma_{1}, \Gamma_{2}\right)$, if the mapping $f:\left(X, \tau_{1}, \tau_{2}\right) \rightarrow\left(Y, \sigma_{1}, \sigma_{2}\right)$ and $g:\left(Y, \sigma_{1}, \sigma_{2}\right) \rightarrow$ $\left(Z, \Gamma_{1}, \Gamma_{2}\right)$ are pairwise weakly fuzzy $\delta$-semi-pre-continuous, then $g \circ f$ : $\left(X, \tau_{1}, \tau_{2}\right) \rightarrow\left(Z, \Gamma_{1}, \Gamma_{2}\right)$ is pairwise weakly fuzzy $\delta$-semi-pre-continuous.

Proof. Let $x_{p} \in X$ and $W$ be any $(i, j)$-fuzzy pre-open subset of $Z$ such that $(g \circ f)\left(x_{p}\right) \in W$. Since $g$ is pairwise weakly fuzzy $\delta$-semi-precontinuous, there exists an $(i, j)$-fuzzy pre-open set $V$ in $Y$ containing $f\left(x_{p}\right)$ such that $V \leq g^{-1}\left((i, j)-\operatorname{spcl}_{\delta}(W)\right)$. Further $f$ being pairwise weakly fuzzy $\delta$-semi-pre-continuous, there exists an $(i, j)$-fuzzy - semi pre-open set $U$ in $X$ such that $x_{p} \in U \leq f^{-1}\left((i, j)-\operatorname{spcl}_{\delta}(V)\right)$. Thus, $x_{p} \in U \leq f^{-1}((i, j)$ $\left.\operatorname{spcl}_{\delta}\left(g^{-1}\left((i, j)-\operatorname{spcl}_{\delta}(W)\right)\right)\right)$. But $g$ being pairwise weakly fuzzy $\delta$-semi-precontinuous, $(i, j)-\operatorname{spcl}_{\delta}\left(g^{-1}(W)\right) \leq g^{-1}\left((i, j)-\operatorname{spcl}_{\delta}(W)\right)$. Therefore, $x_{p} \in$ $U \leq(g \circ f)^{-1}\left((i, j)-s p c l_{\delta}(W)\right)$. Consequently, $g \circ f$ is pairwise weakly fuzzy $\delta$-semi- pre-continuous.

Theorem 3.7. Let $f:\left(X, \tau_{1}, \tau_{2}\right) \rightarrow\left(Y, \sigma_{1}, \sigma_{2}\right)$ be a mapping and $g: X \rightarrow$ $X \times Y$ be the graph mapping of $f$, given by $g\left(x_{p}\right)=\left(x_{p}, f\left(x_{p}\right)\right)$ for $x_{p} \in X$. If $g: X \rightarrow X \times Y$ is pairwise weakly fuzzy $\delta$-semi-pre-continuous, then $f$ is pairwise weakly fuzzy $\delta$-semi-pre-continuous.

Proof. Let $x_{p} \in X$ and $V$ be an $(i, j)$ - fuzzy pre-open set containing $f\left(x_{p}\right)$ in $Y$. Then $X \times Y$ is $(i, j)$ - fuzzy pre-open set in $X \times Y$ containing $g\left(x_{p}\right)$. Since $g$ is pairwise weakly fuzzy $\delta$-semi-pre-continuous, there exists an $(i, j)$-fuzzy $\delta$-semi pre-open set $U$ containing $x_{p}$ in $X$ such that $g(U) \leq$ $(i, j)-\operatorname{spcl}_{\delta}(X \times Y) \leq X \leq(i, j)-\operatorname{spcl}_{\delta}(V)$. Since $g$ is the graph mapping of $f$, we have $f(U) \leq(i, j)-\operatorname{spcl}_{\delta}(V)$. This shows that $f$ is pairwise weakly fuzzy $\delta$-semi-pre-continuous.

\section{Pairwise weakly fuzzy $\delta$-semi pre-open mappings}

In this section, the concept of pairwise weakly fuzzy $\delta$-semi pre-open (preclosed) mapping is to be introduced. Some characterization theorems and basic properties of them are also to be studied.

Definition 4.1. A mapping $f:\left(X, \tau_{1}, \tau_{2}\right) \rightarrow\left(Y, \sigma_{1}, \sigma_{2}\right)$ is said to be pairwise weakly fuzzy- $\delta$-semi pre-open if $f(\lambda) \leq(i, j)$-spint $\delta(f(p c l \lambda))$, for each $(i, j)$-fuzzy open set $\lambda$ of $X$. 
Definition 4.2. A mapping $f:\left(X, \tau_{1}, \tau_{2}\right) \rightarrow\left(Y, \sigma_{1}, \sigma_{2}\right)$ is said to be pairwise weakly fuzzy $-\delta$-semi pre-closed if $(i, j)-\operatorname{spcl}_{\delta}(f(\operatorname{pint}(\lambda))) \leq f(\lambda)$ for each $(i, j)$-fuzzy pre-closed subset $\lambda$ of $X$.

Theorem 4.3. For a mapping $f:\left(X, \tau_{1}, \tau_{2}\right) \rightarrow\left(Y, \sigma_{1}, \sigma_{2}\right)$, the following conditions are equivalent :

(i) $f$ is pairwise weakly fuzzy $\delta$-semi pre-open.

(ii) $(i, j)-\operatorname{spcl}_{\delta}(f(\lambda)) \leq f($ pcl $\lambda)$ for each $(i, j)$-fuzzy pre-open set $\lambda$ of $X$.

(iii) $(i, j)-\operatorname{spcl}_{\delta}(f($ pint $\gamma)) \leq f(\gamma)$ for each $(i, j)$-fuzzy pre-closed set $\gamma$ of $X$.

Proof. $\quad$ (i) $\Rightarrow$ (iii). Let $\gamma$ be a $(i, j)$-fuzzy pre-closed set of $X$. Then $f(1-\gamma)=1-f(\gamma) \leq(i, j)-\operatorname{spint}_{\delta}(f(p c l(1-\gamma))$, by $(\mathrm{i})$.

$\Rightarrow 1-f(\gamma) \leq 1-(i, j)-\operatorname{spcl}_{\delta}(f(\operatorname{pint}(\gamma))$.

Hence $(i, j)-\operatorname{spcl}_{\delta}(f($ pint $\gamma)) \leq f(\gamma)$.

(iii) $\Rightarrow$ (ii). Let $\lambda$ be a $(i, j)$-fuzzy pre-open set of $X$. Since $(i, j)-p c l(\lambda)$ is a fuzzy pre -closed set and $\lambda \leq(i, j)-i n t(p c l \lambda)$, by (iii)

$(i, j)-\operatorname{spcl}_{\delta}(f(\lambda)) \leq(i, j)-\operatorname{spcl}_{\delta} f(\operatorname{intpcl}(\lambda)) \leq f(p c l(\lambda))$.

(ii) $\Rightarrow$ (iii). Let $\gamma$ be a $(i, j)$-fuzzy pre-closed set in X. Since $(i, j)$-pint $\gamma$ is a $(i, j)$ - fuzzy pre-open set and by (ii), $(i, j)-\operatorname{spcl}_{\delta}(f(\lambda)) \leq f(p c l \lambda) \leq$ $f(p c l \gamma)=f(\gamma)[$ since $(i, j)-p c l \gamma=\gamma]$. Therefore $(i, j)-\operatorname{scl} \delta(f($ pint $\gamma)) \leq$ $f(\gamma)$.

(iii) $\Rightarrow$ (i). By (iii), $(i, j)-\operatorname{spcl}_{\delta}(f($ pint $\gamma)) \leq f(\gamma)$ for each $(i, j)$-fuzzy preclosed set $\gamma$ of $X$.

$\Rightarrow 1-f(\gamma) \leq 1-(i, j)-\operatorname{spcl}_{\delta}(f($ pint $\gamma))$

$\Rightarrow f(1-\gamma) \leq(i, j)-\operatorname{spint}_{\delta}(f(p c l(1-\gamma))$

$\Rightarrow f(\alpha) \leq(i, j)-$ spint $_{\delta}(f(p c l \alpha)$

where $\alpha=1-\gamma$ is any $(i, j)$ - fuzzy pre-open set in $X$.

\section{References}

[1] K. Azad, "On fuzzy semicontinuity, fuzzy almost continuity and fuzzy weakly continuity", Journal of mathematical analysis and applications, vol. 82, no. 1, pp. 14-32, Jul. 1981, doi: 10.1016/0022-247X(81)90222-5.

[2] A. Bin Shahna, "On fuzzy strong semicontinuity and fuzzy precontinuity", Fuzzy sets and systems, vol. 44, no. 2, pp. 303-308, Nov. 1991, doi: 10.1016/0165-0114(91)90013-G. 
[3] M. Caldas, S. Jafari and R. Saraf, "Fuzzy $(\delta, \mathrm{p})$ - T1 topological spaces", Journal of Tripura mathematical society, vol. 9, pp. 1-4, 2007.

[4] C. Chang, "Fuzzy topological spaces", Journal of mathematical analysis and applications, vol. 24, no. 1, pp. 182-190, Oct. 1968, doi: 10.1016/0022247X(68)90057-7.

[5] K. Dubey, O. Panwar and R. Tiwari, "On weakly pairwise irresolute mappings", Bulletin of Calcutta mathematical society, vol. 82, pp. 250-255, 1990.

[6] A. Dutta and B. Tripathy, "On fuzzy b- $\theta$ open sets in fuzzy topological space", Journal of intelligent \& fuzzy systems, vol. 32, no. 1, pp. 137-139, Jan. 2017, doi: 10.3233/JIFS-151233

[7] S. Ganguly and S. Saha, "A note on $\delta$-continuity and $\delta$-connected sets in fuzzy set theory", Simon Stevin, vol. 62, pp. 127-141, 1988.

[8] A. Kandil, "On Biproximities and fuzzy bitopological spaces", Simon Stevin, vol. 63, pp. 45-66, 1989.

[9] S. Sampath Kumar, "On fuzzy pairwise $\alpha$-continuity and fuzzy pairwise pre-continuity", Fuzzy sets and systems, vol. 62, no. 2, pp. 231-238, Mar. 1994, doi: 10.1016/0165-0114(94)90063-9.

[10] [S. Sampath Kumar, "Semi-open sets, semi-continuity and semi-open mappings in fuzzy bitopological spaces", Fuzzy sets and systems, vol. 64, no. 3, pp. 421-426, Jun. 1994., doi: 10.1016/0165-0114(94)90166-X.

[11] A. Mukherjee, and R. Dhar, "On weakly fuzzy $\delta$-semi precontinuous mapping and weajly fuzzy $\delta$-semi preirresolute mapping", International journal of fuzzy mathematics, vol. 18, no. 1, pp. 209-216, 2010.

[12] A. Mukherjee and R. Dhar, "On fuzzy $\delta$-semi preopn sets and weakly fuzzy $\delta$-semi preopen functions", Acta ciencia indica, mathematics, vol. 35, no.1, pp. 11- 16, 2009.

[13] A. Mukherjee and A. Dhar, "On pairwise weakly fuzzy $\delta$-semi preirresolute mappings", in Proceedings of international conference on rough sets, fuzzy sets and soft computing. November, 5 - 7, 2009, R. Bhaumik, Ed. Suryamaninagar, TR: Tripura University, 2011, pp. 392-399.

[14] A. Mukherjee and S. Debnath, "On $\delta$-semiopen sets in fuzzy setting", Journal of Tripura mathematical society, vol. 8, pp. 51-54, 2006.

[15] J. Park, "On fuzzy pairwise semi-precontinuity", Fuzzy sets and systems, vol. 93, no. 3, pp. 375-379, Feb. 1998., doi: 10.1016/S01650114(96)00183-2. 
[16] P. Pu and Y. Liu, "Fuzzy topology I. neighbourhood structure of a fuzzy point and Moore - Smith convergence", Journal of mathematical analysis \& applications, vol. 76, no. 2, pp. 571-599, Aug. 1980, doi: 10.1016/0022247X(80)90048-7.

[17] D. Sarma and B. Tripathy, "Pairwise Generalized b-Ro Spaces in Bitopological Spaces", Proyecciones (Antofagasta, En línea), vol. 36, no. 4, pp. 589600, Dec. 2017, doi: 10.4067/S0716-09172017000400589.

[18] S. Thakur and R. Khare, "Fuzzy semi $\delta$-preopen sets and fuzz semi $\delta$-precontinuous mappings", Universitatea din Bacau studii si cerceturi strintitice seria mathematica, vol. 14, pp. 201-211, 2004.

[19] S. Thakur and R. Malviya, "Semi-open sets and semi-continuity in fuzzy bitopological spaces", Fuzzy sets and systems, vol. 79, no. 2, pp. 251-256, Apr. 1996, doi: 10.1016/0165-0114(95)00080-1.

[20] S. Thakur and R. Malviya, Pairwise fuzzy irresolute mappings, Mathematica bohemica, vol. 121, no. 3. pp. 273-280, 1996. [On line]. Available: https://bit.ly/2p90GaE

[21] S. Thakur and S. Singh, "On fuzzy semi-preopen sets and fuzzy semiprecontinuity", Fuzzy sets and systems, vol. 98, no. 3, pp. 383-391, Sep. 1998, doi: 10.1016/S0165-0114(96)00363-6.

[22] B. Tripathy and G. Ray, "On $\delta$ - continuity in mixed fuzzy topologicalspaces", Boletim da sociedade paranaense de matemtica, vol. 32, no. 2, pp. 175-187, 2014, doi: 10.5269/bspm.v32i2.20254.

[23] B. Tripathy and G. Ray, "Weakly continuous functions on mixed fuzzy topological spaces", Acta scienttiarum technology, vol. 36, no. 2, pp.331-335, 2014, doi: 10.4025/actascitechnol.v36i2.16241.

[24] B. Tripathy and S. Debnath, "On fuzzy b-locally open sets in bitopological spaces”, Songklanakarin journal of science and technology, vol. 37, no. 1, pp. 93-96, 2015. [On line]. Available: https://bitly/33qVvDZ

[25] L. Zadeh, “Fuzzy sets”, Information and control, vol. 8, no. 3, pp. 338-353, Jun. 1965, doi: 10.1016/S0019-9958(65)90241-X. 